\title{
EXENTERATION OF THE ORBIT* AND USE OF ALGINATE MOULD FOR APPLYING SKIN GRAFTS
}

\author{
BY \\ JEAN M. DOLLAR AND MARY SAVORY \\ Royal Eye Hospital, London
}

Classical descriptions of exenteration depict it as a swift and sanguinary procedure. Modern methods of anaesthesia and the introduction of diathermy coagulation have made a more exact dissection possible, so that the main interest of the operation now centres in the after-treatment of the cavity. However, it seemed worth while to describe the operation itself, with emphasis on practical details culled from the standard descriptions and from our own experience.

\section{(A) General Considerations}

(1) Conservation or Removal of the Lids. - When it is consistent with the complete removal of all malignant disease, the lids can be conserved, and either turned into the cavity to assist its epithelialization or used to retain a prosthesis. We have not had experience of these procedures, but those who have stress the tendency of the lids to shrink and cause difficulties. Their removal is certainly no obstacle to the fitting of an efficient prosthesis, and simplifies both the operation and the management of the cavity.

(2) Skin Grafting the Cavity.-This is advisable if there are no surgical contraindications. Without it, the cavity granulates, and is slowly epithelialized from the skin margins, meanwhile requiring regular dressings, and producing a discharge which is often offensive and distressing to the patient. The cavity may be grafted immediately, but this involves placing the graft on bare bone, and its blood supply is necessarily precarious. With the aid of antibiotics it is possible to keep the cavity aseptic, and to delay grafting until granulations cover a large part of its walls, usually at the end of 2 weeks. The graft then takes very well.

(3) Method of Applying the Graft.-The success of a skin graft depends mainly on the whole surface being held in contact with the receptor area by firm even pressure. In a hard-walled cavity like the orbit, this is best achieved by making a mould in the cavity. Since the anterior orbital margins overhang its walls, any complete mould must have considerable elasticity if it is to be taken out, draped with the graft, and reinserted. Such a mould can be made from alginates. Sodium alginate is supplied as a sterile syrup-like solution which sets to a jelly on the addition of calcium chloride solution. This jelly is toughened by incorporating in it calcium alginate wool, which resembles ordinary cotton wool. A trial run in a cocktail glass gives a good idea of the behaviour of the materials.

*Received for publication July 7, 1953. 


\section{(B) EXENTERATION}

(1) Incision.-The lids are stitched together. As the skin is easily displaced over the bone, the bony landmarks are palpated, and the line of the desired incision marked out with gentian violet or with dots of diathermy coagulation. Unless the extent of the disease makes it necessary to do otherwise, the line of the incision lies just inside the orbital margin above and below. Medially it lies on the anterior lacrimal crest, and laterally it passes close to the outer canthus. Care should be taken, when making the join between the upper and lower incisions, to avoid carrying the ellipse too far laterally, for when the skin retracts a small triangle of malar bone is exposed. This is very slow to be covered by granulations or skin, and healing is therefore delayed. If the incision round the orbit is too small to allow easy access to the deeper layers, a straight horizontal incision can be made outwards and closed at the end of the operation.

(2) Dissection.-The incision is carried down to the bony margin all round. It is convenient to start at the lower end of the lacrimal crest, to continue the incision below and up towards the outer canthus, and to make the incision above at the last so that blood from it does not obscure the view. Bleeding points are picked up with artery forceps, and coagulated in batches by touching the forceps with the diathermy coagulating terminal. Ligature may be required for a few of the larger arteries.

The periosteum is best incised a few millimetres outside the orbit, rather than on the orbital margin, as the blade of the knife can then be used perpendicularly to the bone. Although the periosteum here is firmly adherent to the bone, it is thick, and does not tear easily when raised. The dissection is begun at the upper outer quadrant, and once between periosteum and bone it is easy to keep in this plane. Freer's nasal raspatories, sharp and blunt, have proved very convenient as they have the right curve for orbital work. After incising the periosteum, the sharp raspatory is used to raise it from the bone, and to separate it as far as the orbital margin. Provided it is not involved in malignant disease, the periosteum strips easily in the orbital cavity using the blunt raspatory and gauze dissection.

The periosteum is adherent at the attachment of the pulley of the superior oblique muscle, and the attachment must be cut, avoiding damage to the roof of the orbit, which may be thin. On the nasal wall of the orbit the periosteum must be raised gently, for the orbital plate of the ethmoid is often very fragile and a sinus may result if the ethmoid air cells are opened. As the periosteal incision is made in the region of the anterior lacrimal crest, the lacrimal sac is reflected laterally with the orbital contents when the periosteum is separated from the nasal wall. The naso-lacrimal duct is identified as the dissection proceeds from the nasal wall to the floor of the orbit. Ligation of the duct has the advantage of preventing ascending infection from the nose, and of stopping blood running down into the nasopharynx. In the floor of the 
orbit periosteal bands passing into the inferior orbital fissure may need to be divided, care being taken to avoid damaging the structures in the fissure. Laterally the temporal and malar branches of the lacrimal artery enter the malar bone, and medially the anterior ethmoidal artery passes from the orbit into the anterior ethmoidal foramen. These vessels are exposed as they pass between periosteum and bone, and if they are clamped and coagulated with diathermy, tiresome bleeding in the depths of the wound is avoided. The periosteum is stripped as far back towards the apex of the orbit as possible.

The shape of the apex of the orbit varies surprisingly, as one finds if one tries possible pedicle clamps in different skulls. It is therefore wise to have a selection of the larger curved artery forceps and to choose the best for use at the time. Most cases require a medial and a lateral clamp, but the exact angle at which they fit best varies. The two clamps are applied as far back as possible, and it is usually advised that two lighter ones be applied in front of them, but the second pair of clamps may tear the periosteum, and make it more difficult to remove the mass cleanly. The pedicle is divided in front of the deep pair of clamps with curved scissors. One is sometimes disappointed at the bulk of tissue left at the apex. After removal of the orbital contents it may be possible to apply clamps behind those previously used, and to reduce the size of the stump by the removal of more tissue. The pedicle is transfixed and ligated. The orbital walls are examined for any evidence of neoplastic involvement, and any diseased bone is chiselled away, cutting through healthy bone. If bony involvement is suspected it may be helpful to have the co-operation of an E.N.T. surgeon.

\section{(C) Management of the Cavity}

At the end of the operation the cavity is dusted with penicillin powder, lined with tulle gras, and packed with vaseline gauze to which penicillin powder is added. It is important to turn the skin edges in towards the cavity, and to extend the pack over them so that they are firmly pressed against the bone. Broad strips of elastoplast over the dressings help to prevent the skin retracting. Patients have remarkably little pain after exenteration, presumably because all sensory nerves to the area have been cut. The dressings are left undisturbed for a week, when the cavity is inspected and repacked as before, usually (for the patient's peace of mind) under general anaesthesia. At this dressing it is usually possible to decide when the cavity can be grafted; our cases have been ready 2 weeks after exenteration.

(1) Skin-Grafting the Cavity using an Alginate Mould.-The mould is prepared in the orbit immediately before cutting the skin graft. The cavity is lined with a square of tulle gras large enough to project over the bony margins. A teaspoonful of the syrup-like alginate is poured in, and pledgets of calcium alginate wool are puddled into this until saturated. Calcium chloride is dripped on to set the mixture, and more sodium alginate and wool are worked in before setting is complete, so as to avoid planes of cleavage. The mould is thus built up by a continuous 
process, and the materials are firmly pressed into the periphery of the orbit to fill it accurately. A handle is built into the centre of the mould and projects on the surface. The handle we are at present using is shown in Fig. 1 ; it consists of a rubber button, threaded with No. 1 black silk; the silk is threaded through a length of fine rubber tubing, which is stiffened by inserting a throat swab stick. The mould is made to fill the orbit, and to overlap its margins for about $2 \mathrm{~cm}$. all round, rising to a dome in the centre. Gentle pressure is thus applied to the whole mould by the dressings. A gentian violet mark is made across the mould and adjacent skin as a guide for reinsertion (Fig. 2). The mould is left in the orbit to consolidate while a Thiersch graft is taken, usually from the inner side of the thigh. The type of Blair's knife with a renewable Gillette blade is convenient for those who do not often cut grafts, as a new blade ensures a perfect edge for each case. Though it is

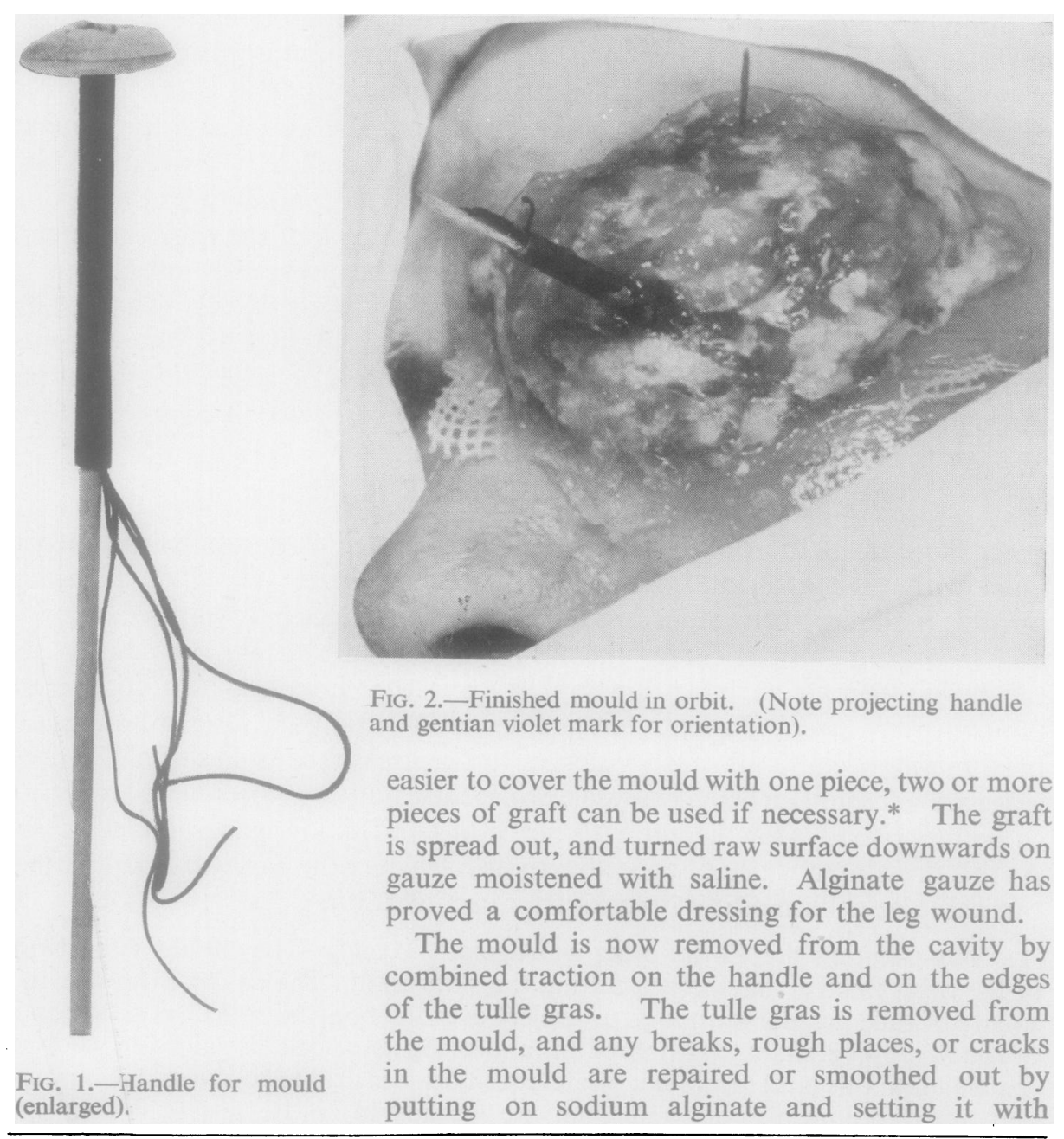

* In one case, where it was necessary to use two pieces of graft, the patch was successfully attached to the main graft by using thromoin and fibrinogen as a physiological glue. 
calcium chloride. The graft is now draped over and carefully spread out on the mould* (Fig. 3). Moistening the inside of the cavity with fibrinogen and the graft with thrombin immediately before inserting seems to improve the take, but is not essential. The mould is put back into the cavity in exactly its original position, and is pressed firmly home. The skin graft covering the mould is thus held in contact with the whole raw area, and no suturing of the edges is

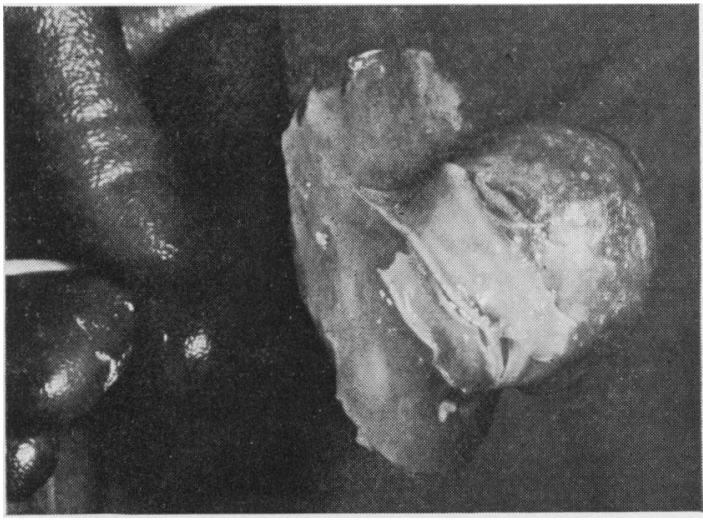

FIG. 3.-Mould draped with skin graft. necessary. The mould is covered with a layer of oiled silk or jaconet to prevent drying, and a pressure dressing of cotton wool and elastoplast applied. The handle is now cut short so that it does not protrude beyond the wool.

(2) After-Treatment.-The mould is removed a week later. The graft now has a milky appearance, which is normal, as its superficial layers usually undergo necrosis, and are shed while the deeper layers survive. There is a frill of dead graft which has overlain the skin round the wound, this is gently cut away, together with any folds of obviously redundant graft. The cavity is lightly packed with vaseline gauze. Hereafter dressings do not need an anaesthetic, and can generally be done at weekly intervals. A change is soon made to dry gauze, as the vaseline makes the skin sodden. Scabs may form in places, and if necessary separation can be hastened by liquid paraffin, or hydrogen peroxide. A prosthesis is fitted as soon as the cavity is healed.

(3) Prosthesis.-Two types are available, and the choice will depend upon the patient's mentality and occupation, and upon the shape of the face.

(a) Spectacle-Attached Prosthesis.-In this type the prosthesis is attached to the spectacle frame, and moves on and off with it. In presbyopic patients the good eye must be fitted with a bifocal lens to avoid the embarrassment of exposing the empty cavity when changing glasses. The occupation must not be one in which glasses are a nuisance, or are likely to be dislodged (Fig $4 a$ and $b$ ).

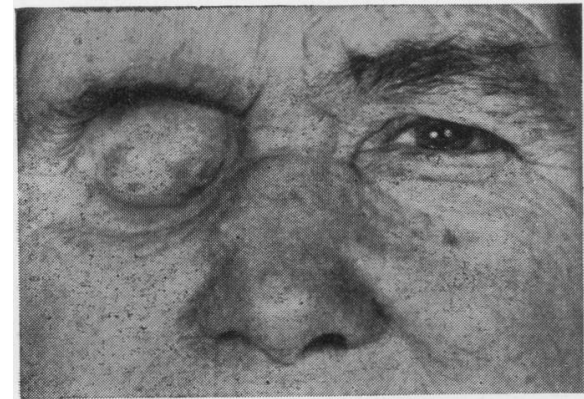

FIG. 4 (a).-Patient without prosthesis.

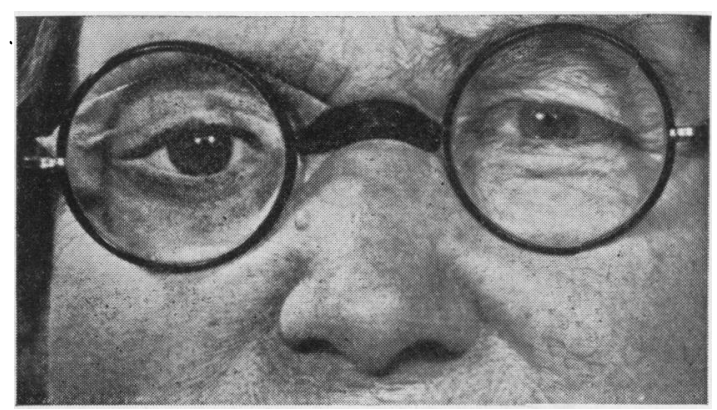

FIG. 4 (b).-Same patient with spectacle-sttached prosthesis. 
(b) Skin-Attached Prosthesis.-This form fits into the socket, and is stuck to the skin margin by a special non-irritant adhesive; it is removed at night. Any spectacle frames should be made by the prosthesis maker so that they give the best possible effect with the prosthesis. With this type a bifocal lens is not essential and the cosmetic effect is usually better than with the first type, specially if make-up and glasses are worn. The patient, however, must be dextrous enough to apply the prosthesis, and must have sufficient vision in the good eye to do so without a correcting lens (Fig $5 a$ and $b$ ).

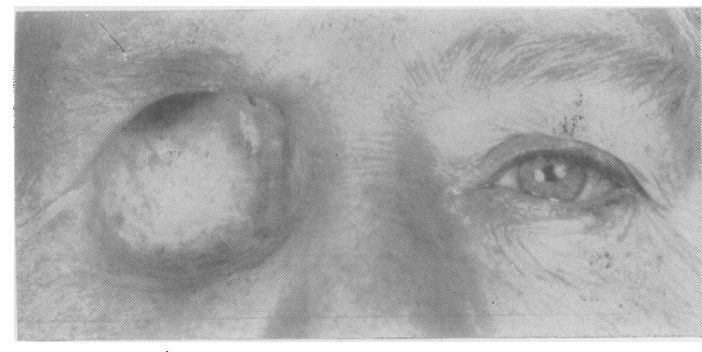

FIG. 5 (a).-Patient without prosthesis.

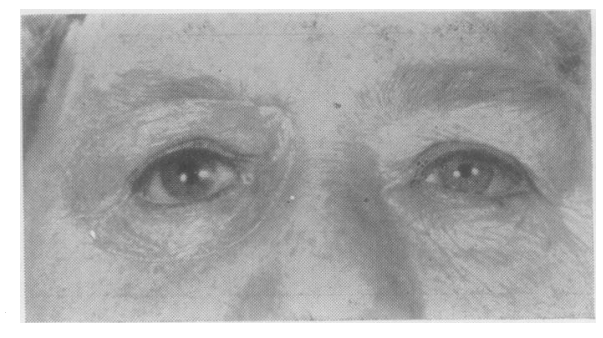

FIG. $5(b)$.- Same patient with skin-attached prosthesis.

\section{Discussion}

As an operation for malignant disease, exenteration must be regarded as an unsatisfactory procedure. There is often only a small margin of normal tissue present round the tumour, and the lymph drainage of the area cannot be removed. It may, however, be the best one can do and it can be useful as a palliative measure for pain or impending fungation. This was the case in two of our patients:

1. A child of five, had an early recurrence after two courses of radio-therapy for a rhabdomyosarcoma of the orbit, and pain was very severe. The operation controlled the pain and fungation until shortly before his death 2 months later.

2. This patient had a blind painful eye, with increasing proptosis, and was found to have extensive retrobulbar extension from a malignant melanoma of the choroid. In spite of this she made a good recovery and the socket healed well. She is now, $2 \frac{1}{2}$ years later, suffering from intrathoracic secondaries, but has had no trouble or discomfort with the socket.

In three other cases, a malignant melanoma of conjunctiva, a small extra-ocular extension from malignant melanoma of choroid, and a mixed tumour of the lacrimal gland (associated with a blind and disfiguring eye), there is more hope that the operation may have been curative, though the follow-up is so far only short; $3 \frac{1}{2}$ years, $2 \frac{1}{4}$ years, and 1 year respectively. The four surviving patients have all been fitted with satisfactory prostheses, and have been cheerful and unconcerned about their deformity.

This alginate mould has proved very satisfactory in use. These substances have obvious advantages over "Stent ", which is a difficult material to sterilize and handle, and which lacks elasticity; they also have the following advantages over the usual dental impression materials: they are supplied 
sterile, and can be resterilized easily; no special temperatures, or measurements of quantities are needed; the mould does not shrink appreciably under the conditions described; breaks, cracks, and irregularities (e.g. those due to the tulle gras), can be repaired at once by using sodium alginate syrup and setting it with calcium chloride.

It is also easy to incorporate a handle into the mould, and to make a mould which completely fills the socket and covers its edges. When the skin graft is draped over it and the mould reinserted, the whole surface of the graft can be firmly pressed against its bed, thus ensuring the most favourable condition for taking.

\section{Summary}

An account is given of practical details in the operation for exenteration of the orbit. The advantages of using an alginate mould for applying the skin graft to the socket are stressed and the technique described in some detail.

The alginate materials described are marketed by Medical Alginates Ltd., Wadsworth Road, Perivale, Middlesex.

The prosthesis shown in Fig. 4 (b) was made by Mr. Brook at Queen Mary's Hospital, Roehampton, under the Ministry of Pensions.

The prosthesis shown in Fig. 5 (b) was made by Mr. Warren, of Clement Clarke Ltd., 16, Wigmore Street, W.1. 